\title{
IMMERSIVE VISUAL EXPERIENCE FOR WAYFINDING ANALYSIS
}

\author{
F. Bianconi ${ }^{1}$, M. Filippucci ${ }^{1}$, F. Cornacchini ${ }^{1 *}$, M. Seccaroni ${ }^{1}$
}

\begin{abstract}
${ }^{1}$ University of Perugia, Italy - fabio.bianconi@unipg.it marco.filippucci@unipg.it filippo.cornacchini@studenti.unipg.it marco.seccaroni@unipg.it
\end{abstract}

\section{Commission II}

KEY WORDS: immersive-reality, wayfinding, eye-tracking, biosensors

\section{ABSTRACT:}

The research presents a new digital approach to wayfinding analysis in immersive reality, integrated with biosensors for the interpretation of data surveyed. The three-dimensional model of the actual state of the Hall of Perugia Railway Station was realized, considering and detecting the present signage, and it was developed a specific algorithm for a multi-platform graphics engine, able to register an interaction mediated by HMD devices. Wayfinding experiences in immersive reality are developed guaranteeing to the user to move freely to complete the missions assigned. The algorithm in this phase runs in the background and records the movements of users and their pupil, saving them in a special file. In the second phase, instead, the real data processing and the subsequent representation of the collected information take place. Starting from its current state, the Station hall is transformed inserting different elements, derived by the aim of developing four scenarios, to analyse different users' response in wayfinding. A three-dimensional heat map of visual attentions is created and the different scenarios can be valued for their impact in the user wayfinding, the results are then confronted with the neuro-analysis obtained by EEG instruments and biosensors. In this way, it is possible to value the impact of the environment and spatial orientation cues, by quantifying and qualifying the impact of the project choices.

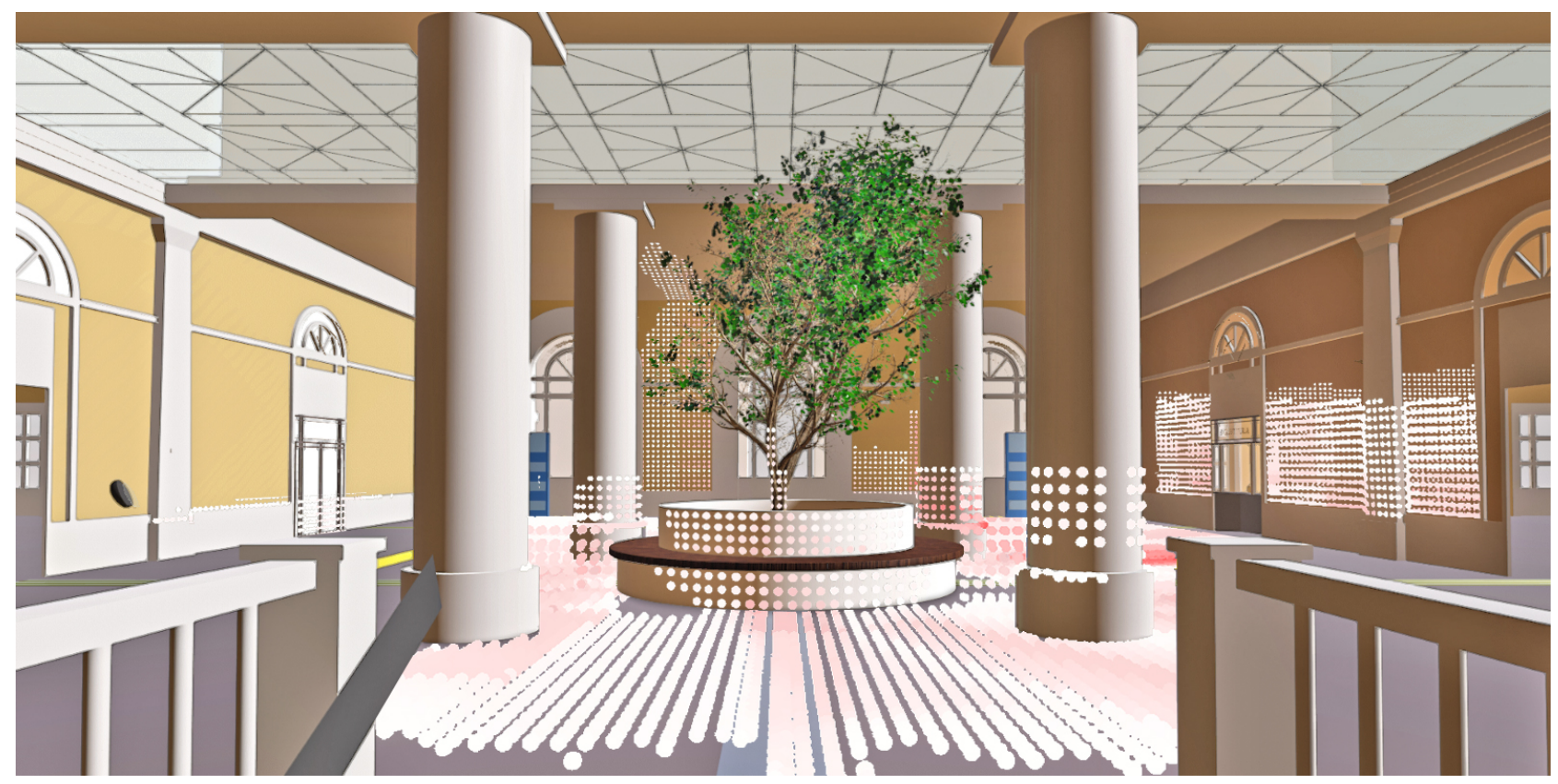

Figure 1. Heat map produced by the algorithm.

\section{INTRODUCTION}

\subsection{Research coordinates}

Wayfinding is a concept attributed to Kevin Lynch (Lynch, 1960) to indicate the study of cognitive processes in reading and orientation in the city, an approach that mixes architecture, urbanism, semiotics and psychology, in the legibility of the environmental complexity in which one is inserted (Alexander, 1964; Bridgman, 1959; Venturi, 1967). In moving through an environment the positions of the surrounding objects with respect to the body constantly change, affecting the construction of updated mental representations that alternate between the projection of the self and the identification of allocentric systems centered on spatial polarities (Wolbers et al., 2008). The wayfinding studies are aimed at the definition of signage system supporting the identification and orientation processes in the urban landscape.

The value of these studies are close to the current culture that has led the visual to be the primary form of approaching and understanding the world to the detriment of the textual (Barnard, 1998; Mirzoeff, 1999), from which it follows that promoting urban wayfinding is one of the key tasks of public spaces (Carr, 1992; Castells, 2008; Gehl, 2007; Goheen, 1998), by virtue of their role in providing services to citizens by enhancing the qualities of places and cities (Gehl and Gemzøe,

\footnotetext{
${ }^{*}$ Corresponding author
} 
2003; Lynch, 1984; Schultz, 1987; Smardon, 1988). Fostering orientation within a space is first and foremost a functional need (Passini, 1981), especially in certain public places where people walk and need information (Belardi and Bianconi, 2012; Devlin, 2014; Symonds, 2017), where, without clear signage, they would lose their statutory purpose. Moreover, a legibility of space (Weisman, 1981) has positive consequences on the interpretation of the place and so on the feelings that the same produces on those who live it (Johnson, 1987), offering security on people and the city itself, avoiding the loss, influencing instead the perception of that feeling of home that determines a well-being (Goldhagen, 2017). The visual experience, in fact, under its apparent immediacy often hides forms of persuasion, which in the case of wayfinding are not limited to directing a behavior (Passini, 1984), that is following the indication (Calori, Chris et al., 2015), but are both means and message (McLuhan, 1967) of the qualities of a place (Lynch, 1984; Mitchell, 1995), by virtue of those processes that succinctly can describe the self-evidence through techniques of classification, separation, and aestheticization (Mirzoeff, 2020).

By reason of visual culture (Jenks, 2002), the wayfinding analysis aims to identify what attracts the eyes (dos Santos et al., 2015), looking for a quantification of the visual exploratory process and then its representation, analyzing in parallel the impact of the images in the mind. The discovery of space can now be investigated in the goal of a survey of the immaterial (Bianconi et al., 2020b, 2020a, 2019b; Bianconi and Filippucci, 2018a; Filippucci, 2019), of what concerns the seeing and the impact of images.

Traditional wayfinding analysis use empirical approaches, registering ex post, in an indirect way, the user experiences (Anderson et al., 2006; Lynch, 1962; Passini, 1981; Picazo et al., 2020; Sancar, 1986) aims at analyzing the impact of the environment (Sharma et al., 2017a) and signage (Calori, Chris et al., 2015; O'neill, 1991; Vilar et al., 2014).

New tools available from neuroscience innovate the empirical analysis, registering human perception and subjective experience in real-time (Banaei et al., 2017). The impact of the environment and spatial orientation cues are analyzed through digital systems proper to neuroscience (Ferlaino, 2019; Goldstein, 2007; Hughes, 2006; Ritchie, 2021; van Schaik, 2013), recorded by using special instrumentation as eyetracking (Chynal et al., 2016; Courtemanche et al., 2018; Crosby and Hermens, 2018; dos Santos et al., 2015; Duchowski, 2017; Hollander et al., 2020; Huddleston et al., 2018; Schiessl et al., 2003; Simpson et al., 2019) to identify what is most attractive in vision. In parallel, EEG helmets are used (Aspinall et al., 2015; Banaei et al., 2017; Boutani and Ohsuga, 2013; Mavros et al., 2016; Pfurtscheller and Lopes Da Silva, 1999; Roe et al., 2013; Sharma et al., 2017b) to record brain frequencies interpreting the mood and the emotions of the tester. Additional biosensors (Sanchez-Comas et al., 2021; Tiwari and Agarwal, 2021; Vahey and Becerra, 2015; Villarejo et al., 2012) may contribute to the interpretation of visual experience (Chatterjee and Vartanian, 2014).

Thus, in general, there is no such thing as a "neutral" environment in an urban context: from a well-being perspective, the anthropogenic environment brings benefits or discomfort (Goldhagen, 2017). Identifying the relationship between the environment and the subject is possible through introduction of the atmosphere (Böhme, 1995). This intangible relationship can be investigated through the use of biosensors such as EEG and Eye-tracker (Li et al., 2016). Through these data and interpreted through a mathematical model that allows through algorithms to go from raw data to cognitive states and then to emotions (Ramirez and Vamvakousis, 2012).
The correlation between data inside the instruments offer behavioral analysis (Chynal et al., 2016; Crosby and Hermens, 2018). These are useful to understanding architectural space value, with a view of opening up to mood detection (Seccaroni et al., 2021) and the design of forms (Bianconi et al., 2019a) around the person (Srivastava et al., 2012).

The analyses can be developed in the real or virtual environment, a field of experimentation (Wilson and Soranzo, 2015) where it is possible to analyze the complexity of the space also in multiple scenarios (Picazo et al., 2020), transforming information in spatial attributes. In fact, the analysis derived by the geometries make it is possible to calculate this relation, "quantifying" perception, through the geometric intersection of an observer-centered visual cone with the digital meshes of the model. Computer analyzes the visual experience, transforming the spatial representations through this information that change during the paths according to distance and field of view implicitly chosen by the user.

Defined the differences and the congruences comparison between experiences in real and digital (Meng and Zhang, 2012), virtual reality strength are linked to the possibility of testing the impacts of possible scenarios also not realized, proposing it as a replication and substitution of the real space (Bielik et al., 2015; Kuliga et al., 2015). Transforming visual experience in analytic data can generate those information useful for the knowledge that guide the wayfinding project.

\subsection{Experimentation objectives}

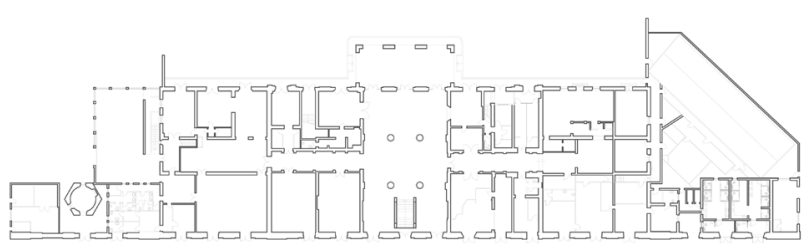

Figure 2. Planimetry of Fontivegge Station.

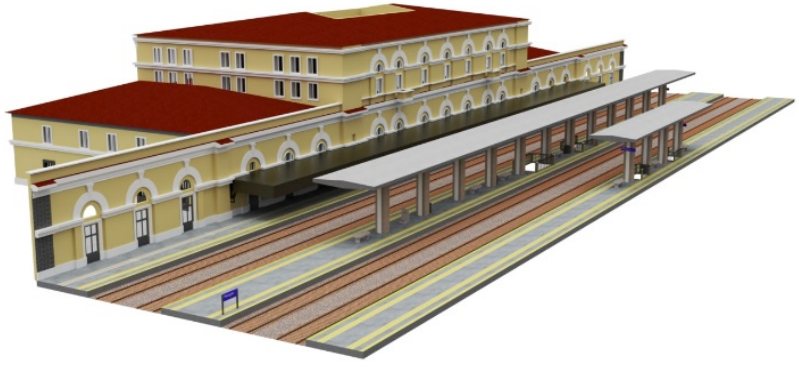

Figure 3. Three-dimensional model of Fontivegge Station.

Inside the wayfinding studies, the project aims to replace eyetracking tools through an algorithm developed within digital exploration environments. Usually eye-tracking analyzes the fixations and finds in correspondence an image of what is projected by the spatial, identifying in the two-dimensional digital representation the correspondence of the fixations. This solution has the randomness of the absence of biunivocity, inherent in the reduction of one of the spatial dimensions. Moreover, this approach does not take into account an influence of the visual field.

A complex programming based on a gaming logic projected towards a serious game, defines algorithms in immersive reality allowing envisaging, in real time, the exploration of space. The 
programming analyzes the user view and its orientation to identify instantaneously his visual cone. This intersects the reconstructed space that is fully coated by a sort of virtual sensors of control, create by a specific algorithm of space discretization, which defines spheres, it can count the intersections in the paths during immersive experiences of different users and represents finally the attraction of every space through an heat map of points regarding the visual attraction.

In order to find a significant case study, the area of the Perugia railway station was chosen, a place of interchange between rail transport, the city's light rail (Minimetrò) and the bus terminal. It is a pole affected by many changes that has led to a chaotic and discontinuous situation, this reflects on the behavior and orientation of users and it is currently involved in a project of urban regeneration (Bianconi and Filippucci, 2018b). The wayfinding analysis are developed with the aim to address spatial transformations inside the system of projects of regeneration plan.

Represented as a digital clone the station (fig. 2-3), different users are involved in a virtual experience where they have to respond to missions, linked to orientation choices. The algorithm in background records user's movements and the variation of point of view, saving firstly data in a special file. It creates a real data processing and a representation of the collected information in the same generative space. The different experiences are then interpolated between them in order to statistically define the attribution of values and heat maps of wayfinding synthesis.

Finally, the use of neural tools aims at interpreting the maps created. The objective of this last phase is the transformation of data in information, essential elements in the environmental knowledge useful for the wayfinding project. In fact, through the results of EEG and galvanic skin response (GSR) data of all the user experiences, it is possible to understand the relationship between the vision map differences and feelings experienced, to evaluate, in the virtual field, the impact of different choices.

For these reasons, in this demonstrative phase, the impacts of four different scenarios are evaluated, comparing the current state (scene 1) with the transformation of visual cues (scene 2), the insertion of benches (scene 3 ) and finally of a tree (scene 4), according to a biophilic design.

\section{MATERIALS AND METHODS}

\subsection{Modelling: scenario, software, details}

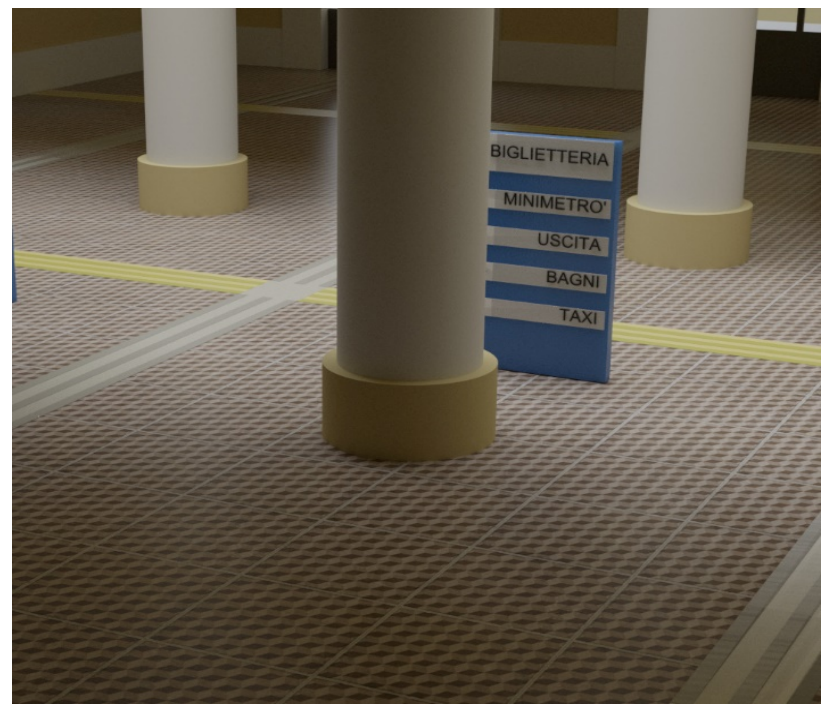

Figure 4. Detail of the three-dimensional model.
The Fontivegge Station in Perugia, chosen as a case study, has been reproduced in a $3 \mathrm{D}$ object created with the mesh modeling software Blender (fig. 4). The level of detail of the reproduction does not aim at photorealism, but is attested to a medium level, with the objective of making the scenario recognizable and easily navigable, without consequences on the fluidity of the experience. In addition to the model of the current state of the station, variations have been created to distinguish the four different areas of the exploration: signs with indications about the places of the station, benches and a flowerbed containing a tree to be placed in the center of the lobby have been created and placed.

\subsection{Generative algorithm of surface discretization}

The created model was imported into Rhinoceros software and an algorithm was developed using the Grasshopper plugin.

The script can be divided into three parts, the first one allows the import and orientation of the UV of the surfaces, the second one allows the division of the surfaces through a network of equidistant points according to pitch that can vary depending on the needs. There is also an approximation, in fact it is not always possible to divide all surfaces by the same length. The last part allows the export in CSV of the list of points divided by surfaces and formatted so that Unity can use it within the scenarios (fig. 5).

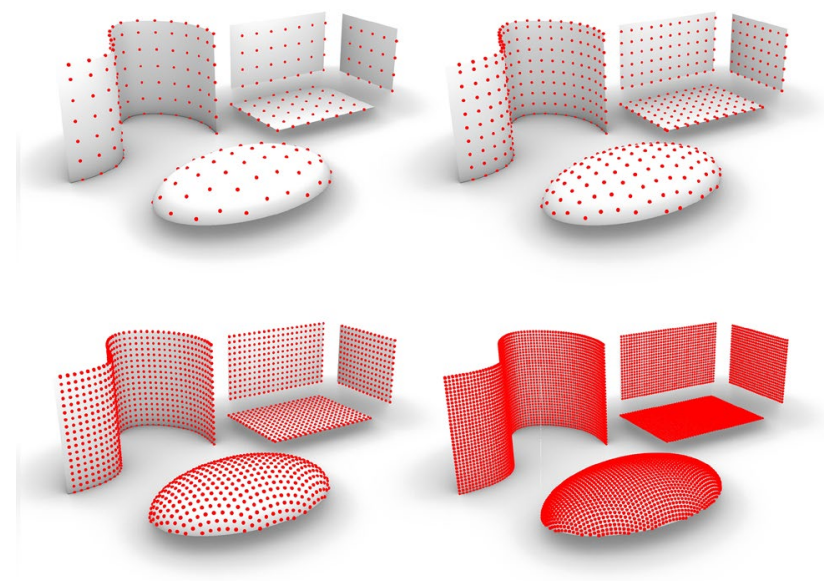

Figure 5. Discretization of surfaces by equidistant points.

\subsection{Level design and illumination}

To make the scenario interactive, it was chosen to develop the programming inside Unity environment as game engine. The three-dimensional model was imported via the fbx exchange format and inserted into the scene. The same process was applied to the variations, those define the different scenarios, and once deactivated, they were inserted into the scene to be activatable according to their use.

In a following phase, the materials have been assigned to the props and the lights have been set. Concerning the materials, simple shaders have been realized using the Universal Render Pipeline (URP), which is a template of the rendering engine that allows obtaining a satisfying graphic rendering without influencing in a heavy way the execution performances. Regarding, instead, the lighting of the scene it was chosen to proceed with a GI (Global Illumination) completely in real time, thus avoiding the process of "baking" of the lightmaps, as the low number of vertices contained in the model allows anyway 
to obtain a smooth execution of the immersive experience on a medium level notebook.

\subsection{Programming interactivity and VR}

The first part of the programming has been dedicated to the code necessary to obtain the interactivity: in fact, it has been realized a simple interface of selection of the scenario to be tested, that in an automatic way appropriately configures the scene, thanks to specific functions activated by the buttons. Subsequently, it has been realized the part of code related to the movements of the user in the virtual scene, both through mouse and keyboard, and through a head mounted display, in this case an HTC VIVE. This head mounted display has 6DOF locomotion capabilities in 3 by 3 meters range and the two external tracking stations can track it. With this technology, users can move freely and look around, interacting with the IVR environment via the two hand controllers. The horizontal FOV of the HTC VIVE is 110 degrees, while the peripheral vision of the human eye is 60 degrees for the near peripheral, 120 degrees for the mid-peripheral and 220 degrees including the far peripheral area.

\subsection{Programming "exploration" level}

Regarding the programming of the immersive virtual experiences and the related data processing, two "game" levels (fig. 6) have been realized completely identical in geometries but with different and complementary functions: the first level is created to allow the virtual experience to users, selecting the appropriate scenario through the previously mentioned interface. Subsequently, it is possible to run the level through which start processing the data collected during the exploration. This separation was necessary because the possible contemporaneity of the experience and information processing would have caused a high number of operations to be performed for each frame of the reproduction, leading to a significant slowdown even on PCs with high computing power.

Describing gradually the characteristics of the levels, it is possible to start from the logic of level one, named "Exploration": once the experience is started, the function for the appearance of the interface for the selection of the scenario with 4 buttons, one for each possibility, is automatically executed. Pressing one of the available buttons will activate the connected function that will configure the props, activating and deactivating the geometries and positioning them appropriately in the scene.

Once the scene is prepared, the interface is removed, the $3 \mathrm{D}$ rendering of the camera corresponding to the user's view is started and the navigation is enabled through the HMD controllers or mouse and keyboard inputs. At this point, the user has the freedom to move freely in the scenery and rotate the inclination of his view. At the same time, the recording of the visitor's interaction with the scene begins: at each frame, in fact, a function is executed to record on a list of variables the cartesian coordinates $(\mathrm{x}, \mathrm{y}, \mathrm{z})$ related to the position in the scene of the user, the three coordinates of the rotation that identify the angle of the camera and then of the view and a time reference of the moment in which the data is collected.

At the same time, the recording of the visitor's interaction with the scene begins: at each frame, in fact, a function is executed to record on a list of variables the same cartesian coordinates relative to the position in the scene of the user, the three coordinates of the rotation that identify the angle of the camera and, therefore, of the view and a temporal reference of the moment in which the data is collected. From this moment on, the recording will be performed automatically until, through an input, the immersive experience is interrupted. With the stop of the playback the final function is activated to save the data collected in the list on a text file in csv format on a folder of the $\mathrm{PC}$ selected by the user in an initial phase of configuration of the algorithm.

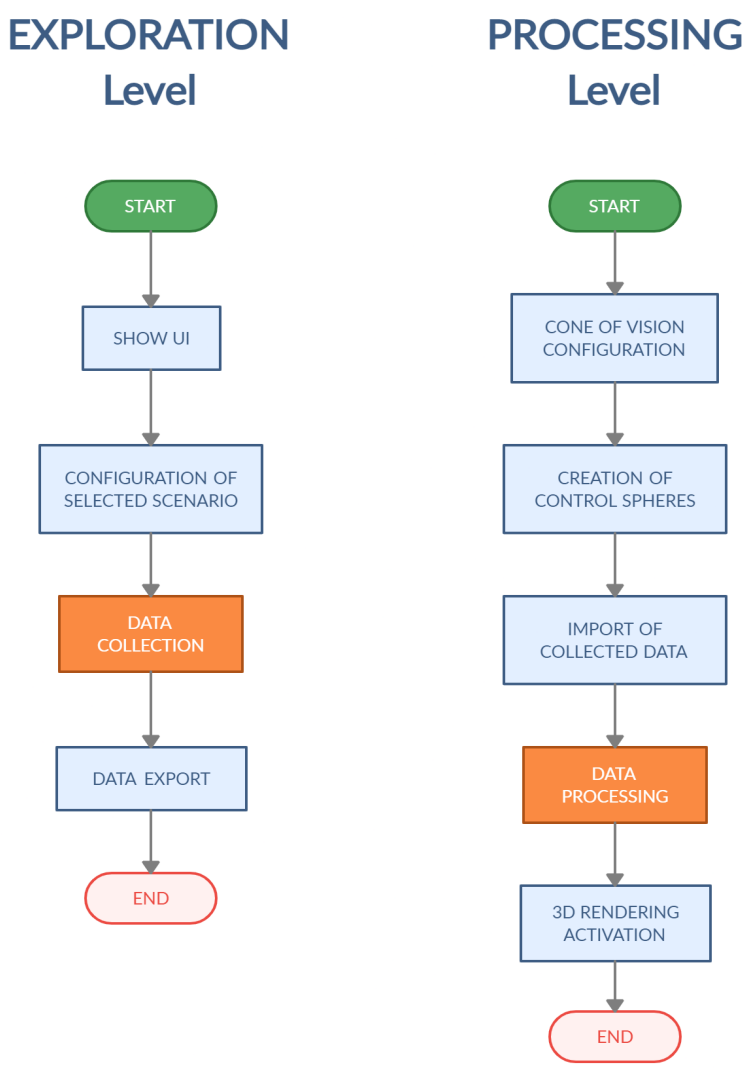

Figure 6. Flow chart of the two level logic.

\subsection{Programming "processing" level}

The logic of the second level, called "Processing" begins with a configuration phase of the visual cone to be used in the data analysis phase. In the configurations, it is possible, to customize some parameters related to the shape of the cone that will be projected, including the opening angle and the height, which determines the depth of the visual field, related to the hypothetical user speed. Starting the processing, then, an algorithm collects this information and applies it to the geometry of the cone to resize it. Anyway, the field of view and the cone are not limited in his height, hypothesizing that the visual experience is lived in closed spaces.

Subsequently the algorithm retrieves the csv file exported by Grasshopper and, for each point indicated, instantiates in the scene a small sphere that will serve to intercept the projection of the visual cone. To the single spheres, in fact, it has been associated a small script able to recognize the collisions with the geometry of the cone and to memorize in an appropriate variable the times that the contact with it has happened.

The next step is to retrieve and process the csv file processed in the previous level: the algorithm is configured to read the records of the file and, for each line, project the cone from the saved point $\mathrm{x}, \mathrm{y}$, and $\mathrm{z}$, and orient it according to the coordinates of the rotation. By repeating this procedure for all the annotated coordinates, the control spheres are able to record the number of overlaps with the cone and with, therefore, how 
many times the virtual visitor's eye has intercepted the specific portion of the $3 \mathrm{D}$ model. In this phase the three-dimensional rendering is deactivated, since this would have led to an increase in the number of calculations and therefore to an unnecessary use of computing power. The algorithm, however, through control messages, informs the operator about the operations in elaboration, describing the phases in progress and through a counter of the calculations made, it compares the total number of the operations.

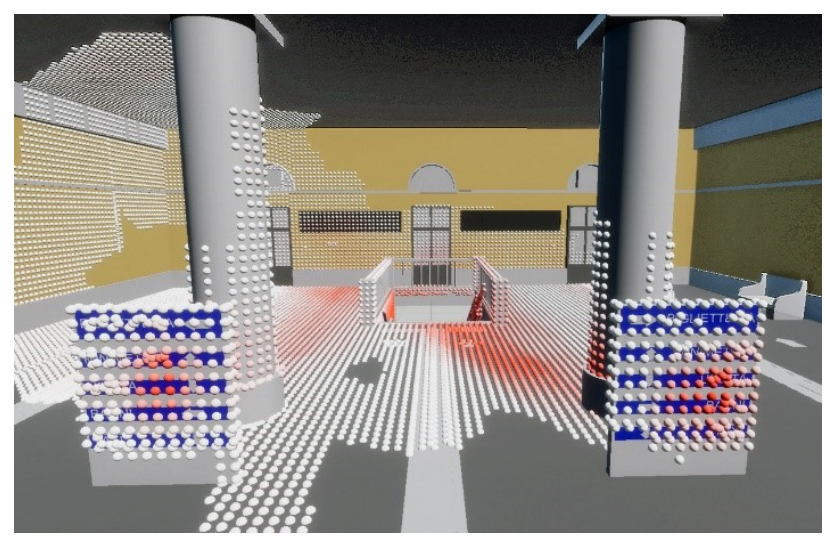

Figure 7. Three-dimensional representation of the results.

Once the analysis procedures are finished, the logic of the level proceeds with the coloring part of the created spheres (fig. 7). The sphere with the highest number of interceptions with the cone is identified and a color scale is created assigning full color (e.g. red) to that sphere, while for the others the coloring is gradually tending to white, always in relation to the number of contacts recorded. The spheres that have not recorded contacts are completely hidden in order not to make the model too heavy, from both the point of view of the rendering elaboration and of the aesthetics.

Ended the elaboration part, the algorithm launches the rendering of the scene, so that the results can be graphically compared. The programming generates two opposite orthogonal axonimeters (fig. 8). A csv file is saved, derived from the one containing the coordinates of the sphere insertion points, to which the value of the reciprocal counter is added for each. In this way, it will be possible to visualize the results of the various elaborations in subsequent phases, simply by selecting one of these files at the beginning of the procedure. Moreover, the navigation functions are activated, to make it possible to move the camera freely in the scene to visualize the results from the necessary point of view.

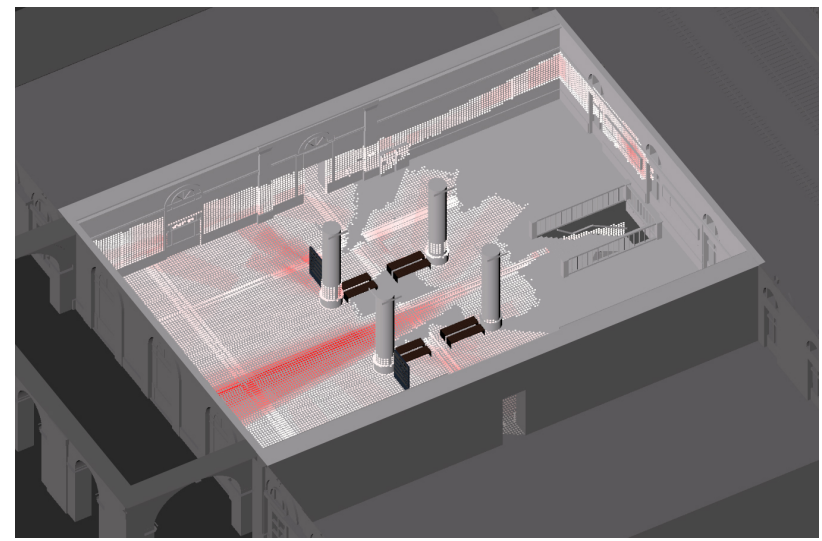

Figure 8. Axonometric representation of the results.

\subsection{Biosensors}

The following data were acquired during the immersive experience:

- EEG (fig. 9)

- GSR (fig. 10)

EEG was acquired using Muse2. This device allows to record 4 channels (TP9, AF7, AF8, TP10) and their frequencies $(\alpha, \beta, \gamma$, $\delta, \theta)$ as well as the timestamp for time synchronization with scenarios and the GRS.

The GRS is an eSense Mindfield, it allows to record the electrical conductance with $5 \mathrm{hz}$ interval as well as the skin conductance responses (SCR). Also, in this case the timestamp is present so as to synchronize absolutely with all other devices. The RAW data of the eeg through an interpretative model it is possible to obtain the emotions felt by the observer.

A further confirmation of these emotion variations is possible through GRS.

The data processed in this way aims to interpret and characterize the heatmaps from the point of view of emotions. The designer outside the algorithmic process with the generated perceptual heatmaps will compare these data.

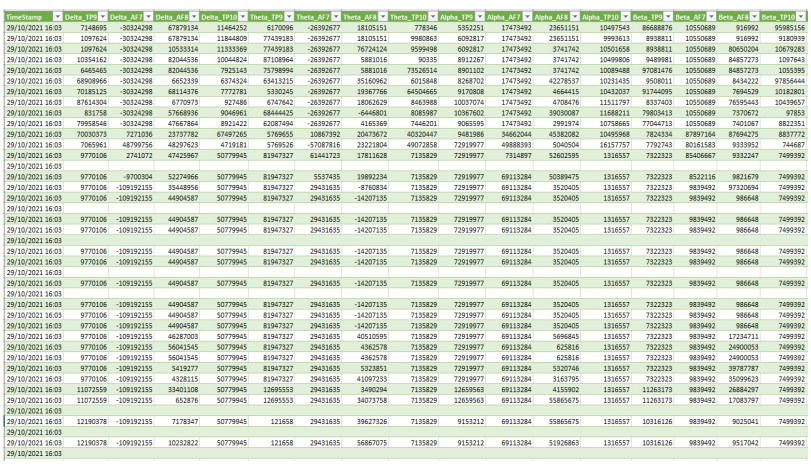

Figure 9. EEG data acquired using Muse2.

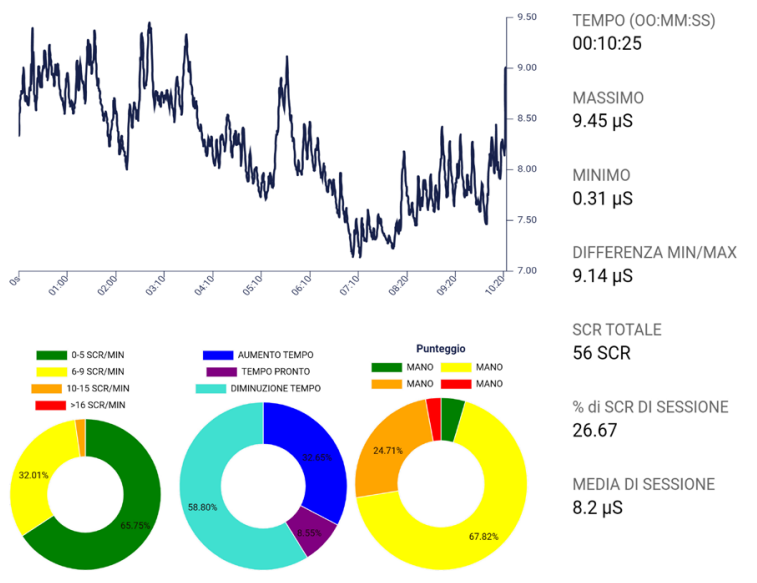

Figure 10. GSR data recorded through eSense Mindfield.

\section{RESULTS}

The algorithm was verified by running tests on all configured scenarios and data were collected simultaneously from the biosensors described above. These data show different results for each tested configuration:

- Scenario 1: is the one related to the current state and testifies a situation of disorientation of the user, also confirmed 
by the data collected with the sensors: it is possible to notice, in fact, how the user has inspected the whole area looking for spatial information, in vain, experiencing on average negative emotional states such as "stress" and "unhappiness".

- Scenario 2: represents the current state with the addition of information totems and it describes shorter experiences, as the user reaches the assigned goal more easily, exploiting, as the heatmaps indicate, the information included in the scene. From an emotional point of view there are no predominant emotions but on average they remain in the negative quadrant.

- Scenario 3: this is the one with the inclusion of the benches in the center of the atrium. This variation does not lead to a change in the passing of the proposed tests, but generates EEG and GSR data which testify to the appreciation for the new configuration; in fact, the emotions experienced remain in the positive quadrant with a characterization of "pleasure" and "serene".

- Scenario 4: the flowerbed with the seats and the tree in the center was added and generated the most significant data: the heatmaps confirm through the full red colored spheres the attraction exerted by the tree during the experience. This interest has produced data from biosensors that certify a positive influence of the inclusion of green, in fact, from the analysis is predominant as an emotion "tranquility".

The results obtained highlight the need to redesign the space and the orientation within information. In addition, the graphics produced by the algorithm highlight areas of the facility that are potentially optimal for inserting new signs or moving present ones.

\section{CONCLUSIONS}

The algorithm developed has proved to be a useful tool for the identification of some of the critical issues related to the orientation in the area of interest and to extrapolate some ideas for the future design of solutions for wayfinding. The mathematization of space inherent in the process of digital representation is proposed as an optimal condition to develop analysis and to quantify the impact of an environment on those who live it, analytical condition that becomes of primary support to design choices, for the ability to quantify in advance the impact of possible assumed choices.

The visual experiences, also in immersive reality, represents the pretext useful for extract relations between different spaces and behaviors, according to the centrality of the eye in our visual culture. Different scenarios, projected to transform the spatial hermeneutics, transforms impacts in the vision and in the place fillings. The enrichment of virtual space modelled with information of visual experiences shows itself as a concrete demonstration of representation of immaterial relations, which define the place quality.

The programmed algorithm opens up to multiple upgrades that concern a different description of the visual interpretation transposed in the attribution of values in correspondence with ranges defined by the degrees of the opening of the visual cone, a different application in the open field or in the detail of the studies of graphic design, a different integration with biosensors.

Wayfinding analyses developed in immersive reality offers the possibility of demonstrating the hypothesis of projecting choices in identification and orientations processes, analyzing the environmental impacts in the eye and in the brain, without involving empirical surveys with their aleatoric selfacknowledgments of the feelings experienced. Digital model becomes in this way the field of experimentations, of variation of architectural design, support in the idea of concretization.

\section{REFERENCES}

Alexander, C., 1964. Notes on the synthesis of form. Harvard University Press, Cambridge.

Anderson, N.S., Norman, D.A., Draper, S.W., 2006. User Centered System Design: New Perspectives on HumanComputer Interaction. Am. J. Psychol. 101, 148 https://doi.org/10.2307/1422802

Aspinall, P., Mavros, P., Coyne, R., Roe, J., 2015. The urban brain: Analysing outdoor physical activity with mobile EEG. Br. J. Sports Med. 49, 272-276. https://doi.org/10.1136/bjsports-2012-091877

Banaei, M., Hatami, J., Yazdanfar, A., Gramann, K., 2017. Walking through architectural spaces: The impact of interior forms on human brain dynamics. Front. Hum. Neurosci. 11. https://doi.org/10.3389/fnhum.2017.00477

Barnard, M., 1998. What is Visual Culture?, in: Art, Design and Visual Culture. pp. 10-31. https://doi.org/10.1007/978-1-34926917-4 2

Belardi, P., Bianconi, F., 2012. I am a hospital. Dal wayfinding al waysharing per il miglioramento della percezione spaziale e dell'orientamento negli ospedali. Alinea Editrice, Firenze.

Bianconi, F., Filippucci, M., 2018a. Icnografie castiglionesi : ricerche e studi per la rappresentazione e il rilievo del patrimonio rurale. Maggioli, Santarcangelo di Romagna.

Bianconi, F., Filippucci, M., 2018b. Rappresentazione, percezione, progetto. Il ruolo dell'Università per Perugia città smart, in: Rappresentazione Materiale/Immateriale - Drawing as (in) Tangible. Gangemi, pp. 37-48.

Bianconi, F., Filippucci, M., Cornacchini, F., 2020a. Play and transform the city. Scires-it 10, 141-158. https://doi.org/10.2423/i22394303v10n2p141

Bianconi, F., Filippucci, M., Felicini, N., 2019a. Immersive wayfinding: virtual reconstruction and eye-tracking for orientation studies inside complex architecture. ISPRS - Int. Arch. Photogramm. Remote Sens. Spat. Inf. Sci. XLII-2/W9, 143-150. https://doi.org/10.5194/isprs-archives-xlii-2-w9-1432019

Bianconi, F., Filippucci, M., Pelliccia, G., Seccaroni, M., Meschini, M., 2020b. New signs for the urban landscape. The Bus Rapid Transit case redesigns the city, in: De-Sign Environment Landscape City. Genova University Press, pp. 4358.

Bianconi, F., Filippucci, M., Seccaroni, M., 2019b. Survey and co-design the urban landscape. Innovative digital path for perception analysis and data-driven project, in: International Archives of the Photogrammetry, Remote Sensing and Spatial Information Sciences - ISPRS Archives. https://doi.org/10.5194/isprs-archives-XLII-2-W15-165-2019

Bielik, M, Schneider, S, Kuliga, S, Valášek, M, Donath, D, Bielik, Martin, Schneider, Sven, Kuliga, Saskia, Valášek, Milan, Donath, Dirk, 2015. SSS 10 Proceedings of the 10th International Space Syntax Symposium Investigating the effect of urban form on the environmental appraisal of streetscapes Investigating the effect of urban form on the environmental 
appraisal of streetscapes. London, pp. 1-13.

Böhme, G., 1995. Atmosphäre: Essays zur neuen Ästhetik. Suhrkamp, Berlin.

Boutani, H., Ohsuga, M., 2013. Applicability of the "Emotiv EEG Neuroheadset" as a user-friendly input interface, in: Proceedings of the Annual International Conference of the IEEE Engineering in Medicine and Biology Society, EMBS. pp. 1346-1349. https://doi.org/10.1109/EMBC.2013.6609758

Bridgman, P.W., 1959. The way things are. Harvard University Press, Cambridge.

Calori, Chris, Vanden-Eynden, David, A., Chermayeff, Ivan, author of introduction, E., Geismar, Tom, author of introduction, E., 2015. Signage and wayfinding design: a complete guide to creating environmental graphic design systems / Chris Calori, David Vanden-Eynden; forewords by Ivan Chermayeff, Tom Geismar.

Carr, S., 1992. Public space. Cambridge University Press, Cambridge.

Castells, M., 2008. The new public sphere: Global civil Society, communication networks, and global governance. Ann. Am Acad. Pol. Soc. Sci. https://doi.org/10.1177/0002716207311877 Chatterjee, A., Vartanian, O., 2014. Neuroaesthetics. Trends Cogn. Sci. https://doi.org/10.1016/j.tics.2014.03.003

Chynal, P., Sobecki, J., Rymarz, M., Kilijanska, B., 2016. Shopping behaviour analysis using eyetracking and EEG. Proc. - 2016 9th Int. Conf. Hum. Syst. Interact. HSI 2016 458-464. https://doi.org/10.1109/HSI.2016.7529674

Courtemanche, F., Léger, P.-M., Dufresne, A., Fredette, M., Labonté-LeMoyne, É., Sénécal, S., 2018. Physiological heatmaps: a tool for visualizing users' emotional reactions. Multimed. Tools Appl. 77, 11547-11574. https://doi.org/10.1007/s1 1042-017-5091-1

Crosby, F., Hermens, F., 2018. Does it look safe? An eye tracking study into the visual aspects of fear of crime. Q. J. Exp. Psychol. 174702181876920 https://doi.org/10.1177/1747021818769203

Devlin, A.S., 2014. Wayfinding in healthcare facilities: Contributions from environmental psychology. Behav. Sci. (Basel). https://doi.org/10.3390/bs4040423

dos Santos, R.D.O.J., de Oliveira, J.H.C., Rocha, J.B., Giraldi, J.D.M.E., 2015. Eye Tracking in Neuromarketing: A Research Agenda for Marketing Studies. Int. J. Psychol. Stud. 7. https://doi.org/10.5539/ijps.v7n1p32

Duchowski, A.T., 2017. Eye Tracking Methodology. SpringerVerlag, London. https://doi.org/10.1007/978-3-319-57883-5

Ferlaino, M., 2019. Neuroarchitecture: quantifying perception to inform a design for improved mental well-being.

Filippucci, M., 2019. Rappresentazione e percezione per nuove strategie di sviluppo territoriale dell'Umbria. Il cibo come riflesso del paesaggio, il paesaggio come riflesso del cibo, in: Riflessioni: L'arte Del Disegno/Il Disegno Dell'arte. Gangemi, pp. 1589-1602.
Gehl, J., 2007. Public spaces for a changing public life. Open Sp. People Sp. 3-11. https://doi.org/10.4324/9780203961827 Gehl, J., Gemzøe, L., 2003. New city spaces. The Danish Architectural Press, Copenhagen.

Goheen, P.G., 1998. Public space and the geography of the modern city. Prog. Hum. Geogr. https://doi.org/10.1191/030913298672729084

Goldhagen, S.W., 2017. Welcome to Your World. How the Built Environment Shapes Our Lives. HarperCollins, New York.

Goldstein, E.B., 2007. Sensation and perception. Thomson/Wadsworth, Belmont.

Hollander, J.B., Sussman, A., Purdy Levering, A., FosterKarim, C., 2020. Using Eye-Tracking to Understand Human Responses to Traditional Neighborhood Designs. Plan. Pract. Res. 35. https://doi.org/10.1080/02697459.2020.1768332

Huddleston, P.T., Behe, B.K., Driesener, C., Minahan, S., 2018. Inside-outside: Using eye-tracking to investigate search-choice processes in the retail environment. J. Retail. Consum. Serv. 43, 85-93. https://doi.org/10.1016/j.jretconser.2018.03.006

Hughes, J.C., 2006. Neuroethics: defining the issues in theory, practice, and policy. J. Neurol. Neurosurg. Psychiatry 77, 568. https://doi.org/10.1136/jnnp.2005.083881

Jenks, C., 2002. Visual culture. Routledge, London

Johnson, M., 1987. The Body in the Mind: The Bodily Basis of Meaning, Imagination, and Reason, The Journal of Aesthetics and Art Criticism. University of Chicago Press, Chicago. https://doi.org/10.2307/431155

Kuliga, S.F., Thrash, T., Dalton, R.C., Hölscher, C., 2015. Virtual reality as an empirical research tool - Exploring user experience in a real building and a corresponding virtual model. Comput. Environ. Urban Syst. 54, 363-375.

Li, B., Wang, Y., Wang, K., 2016. Data fusion and analysis techniques of neuromarketing. WIT Trans. Eng. Sci. 113, $396-$ 404. https://doi.org/10.2495/IWAMA150461

Lynch, K., 1984. Good city form. Harvard-MIT, Cambridge.

Lynch, K., 1962. The Image of the City, The Journal of Aesthetics and Art Criticism. MIT Press, Massachusetts. https://doi.org/10.2307/427643

Lynch, K., 1960. The image of the city. Harvard-MIT , Cambridge.

Mavros, P., Austwick, M.Z., Smith, A.H., 2016. Geo-EEG: Towards the Use of EEG in the Study of Urban Behaviour. Appl. Spat. Anal. Policy 9, 191-212. https://doi.org/10.1007/s12061-015-9181-z

McLuhan, M., 1967. The Medium is the Massage: An Inventory of Effects, Gingko Press. Penguin, London. https://doi.org/10.1177/135050768701800104

Meng, F., Zhang, W., 2012. A review of wayfinding and a new virtual reality system for wayfinding studies. Int. J. Serv. Oper. Informatics

7 ,

197-211. 
https://doi.org/10.1504/ijsoi.2012.051399

Mirzoeff, N., 2020. The Right to Look, The Right to Look. Duke University Press. https://doi.org/10.1515/9780822393726

Mirzoeff, N., 1999. An Introduction to Visual Culture. Routledge 274 .

Mitchell, W.J., 1995. City of bits: space, place, and the infobahn. MIT Press, Cambridge.

O'neill, M.J., 1991. Effects of signage and floor plan configuration on wayfinding accuracy. Environ. Behav. 23, 553-574. https://doi.org/10.1177/0013916591235002

Passini, R., 1984. Wayfinding in architecture.

Passini, R., 1981. Wayfinding: A conceptual framework. Urban Ecol. 5, 17-31. https://doi.org/10.1016/0304-4009(81)90018-8

Pfurtscheller, G., Lopes Da Silva, F.H., 1999. Event-related EEG/MEG synchronization and desynchronization: Basic principles. Clin. Neurophysiol. https://doi.org/10.1016/S13882457(99)00141-8

Picazo, J., Hazenoot, A., Otaduy, C., Braux, M., Bong, W., 2020. User-centred design for a not straightforward university wayfinding, in: Proceedings of the 22nd International Conference on Engineering and Product Design Education, E and PDE 2020. The Design Society. https://doi.org/10.35199/epde.2020.11

Ramirez, R., Vamvakousis, Z., 2012. Brain informatic. IEEE Intell. Syst. 26, 16-21. https://doi.org/10.1109/MIS.2011.83

Ritchie, I., 2021. Neuroarchitecture: Designing with the Mind in Mind.

Roe, J.J., Aspinall, P.A., Mavros, P., Coyne, R., 2013. Engaging the brain: the impact of natural versus urban scenes using novel EEG methods in an experimental setting. Environ. Sci. 1, 93104. https://doi.org/10.12988/es.2013.3109

Sancar, F.H., 1986. Wayfinding in architecture. Landsc. J. 5, 71-73. https://doi.org/10.3368/1j.5.1.71

Sanchez-Comas, A., Synnes, K., Molina-Estren, D., TroncosoPalacio, A., Comas-González, Z., 2021. Correlation analysis of different measurement places of galvanic skin response in test groups facing pleasant and unpleasant stimuli. Sensors 21 . https://doi.org/10.3390/s21124210

Schiessl, M., Duda, S., Thölke, A., Fischer, R., 2003. Eye Tracking and its Application in Usability and Media Research. MMI Interakt. - Eye Track. 1, 41-50.

Schultz, N., 1987. On the Way to Figurative Architecture, Berkeley Planning Journal. https://doi.org/10.5811/westjem.2011.5.6700

Seccaroni, M., Aquinardi, C.M., Bettollini, E., 2021. Urban Atmospheres: Representation of Intangible Relations, in: Lecture Notes in Civil Engineering. Springer Science and Business Media Deutschland GmbH, pp. 1111-1128. https://doi.org/10.1007/978-3-030-59743-6_52

Sharma, G., Kaushal, Y., Chandra, S., Singh, V., Mittal, A.P., Dutt, V., 2017a. Influence of landmarks on wayfinding and brain connectivity in immersive virtual reality environment. Front. Psychol. 8. https://doi.org/10.3389/fpsyg.2017.01220

Sharma, G., Kaushal, Y., Chandra, S., Singh, V., Mittal, A.P., Dutt, V., 2017b. Influence of Landmarks on Wayfinding and Brain Connectivity in Immersive Virtual Reality Environment. $\begin{array}{llll}\text { Front. } & \text { Psychol. } & \text { 8, }\end{array}$ https://doi.org/10.3389/fpsyg.2017.01220

Simpson, J., Freeth, M., Simpson, K.J., Thwaites, K., 2019. Visual engagement with urban street edges: insights using $\begin{array}{llll}\text { mobile eye-tracking. J. Urban. } 12 . & \text { J. }\end{array}$ https://doi.org/10.1080/17549175.2018.1552884

Smardon, R.C., 1988. Perception and aesthetics of the urban environment: Review of the role of vegetation. Landsc. Urban Plan. 15, 85-106. https://doi.org/10.1016/0169-2046(88)900187

Srivastava, M., Abdelzaher, T., Szymanski, B., 2012. Humancentric sensing. Philos. Trans. R. Soc. A Math. Phys. Eng. Sci. 370, 176-197. https://doi.org/10.1098/rsta.2011.0244

Symonds, P., 2017. Wayfinding Signage Considerations in International Airports.

Tiwari, S., Agarwal, S., 2021. A Shrewd Artificial Neural Network-Based Hybrid Model for Pervasive Stress Detection of Students Using Galvanic Skin Response and Electrocardiogram Signals. Big Data 4, 427-442. https://doi.org/10.1089/big.2020.0256

Vahey, R., Becerra, R., 2015. Galvanic skin response in mood disorders: A critical review, International Journal of Psychology and Psychological Therapy.

van Schaik, K., 2013. How neuroscience contributes to neuromarketing. essay.utwente.nl.

Venturi, R., 1967. Complexity and Contradiction in Architecture. The Museum of modern art, New York.

Vilar, E., Rebelo, F., Noriega, P., Duarte, E., Mayhorn, C.B., 2014. Effects of competing environmental variables and signage on route-choices in simulated everyday and emergency wayfinding situations. Ergonomics 57, 511-524. https://doi.org/10.1080/00140139.2014.895054

Villarejo, M.V., Zapirain, B.G., Zorrilla, A.M., 2012. A stress sensor based on galvanic skin response (GSR) controlled by ZigBee. Sensors (Switzerland) 12, 6075-6101. https://doi.org/10.3390/s120506075

Weisman, J., 1981. Evaluating architectural legibility: WayFinding in the Built Environment. Environ. Behav. 13, 189204. https://doi.org/10.1177/0013916581132004

Wilson, C.J., Soranzo, A., 2015. The Use of Virtual Reality in Psychology: A Case Study in Visual Perception. Comput. Math. Methods Med. 2015. https://doi.org/10.1155/2015/151702

Wolbers, T., Hegarty, M., Büchel, C., Loomis, J.M., 2008. Spatial updating: How the brain keeps track of changing object locations during observer motion. Nat. Neurosci. 11, 12231230. https://doi.org/10.1038/nn.2189 